\title{
BMJ
}

\section{Naftidrofuryl for intermittent claudication: meta-analysis based on individual patient data}

\author{
T De Backer, clinical cardiologist, , ${ }^{1,2} \mathrm{R}$ Vander Stichele, research fellow, ${ }^{1}$ P Lehert, professor of statistics, ${ }^{3}$ \\ $L$ Van Bortel, professor of clinical pharmacology ${ }^{1,2}$
}

'Heymans Institute of

Pharmacology, Ghent University, Ghent, Belgium

${ }^{2}$ Department of Cardiovascular

Disease, University Hospital

Ghent, Belgium

${ }^{3}$ Department of Statistics,

Facultes Universitaires

Catholiques de Mons, Leuven

Academy, Mons, Belgium

Correspondence to: T De Backer

Department of Cardiovascular

Disease, University Hospital Ghent,

De Pintelaan 185, 9000 Ghent,

Belgium tine.debacker@Ugent.be

Cite this as: BMJ 2009;338:b603 doi:10.1136/bmi.b603

\section{ABSTRACT}

Objective To assess the efficacy of naftidrofuryl compared with placebo in treating the symptoms of intermittent claudication.

Design Meta-analysis based on individual patient data.

Data sources Medline, International Pharmaceutical Abstracts, Embase, Science Citation Index, and the Cochrane trial registers. Reference lists of retrieved articles were checked. Authors and companies were approached for additional information and individual patient data.

Inclusion criteria Double blind, randomised controlled trials in patients with intermittent claudication receiving oral naftidrofuryl or placebo and with pain-free walking distance as primary outcome.

Data collection Individual patient data were collected from electronic data or from case report forms and checked for integrity.

Analysis All randomised patients were analysed following the intention to treat principle. Efficacy was assessed by the ratio of geometric mean of the relative improvement in pain-free walking distance after use of naftidrofuryl compared with placebo. In the analysis of responders, therapeutic success was defined as an improvement of walking distance at baseline by at least $50 \%$.

Results In total, 1266 patients were randomised (1083 in the main analysis). The ratio of relative improvement in pain-free walking distance after use of naftidrofuryl compared with placebo was 1.37 (95\% confidence interval 1.27 to 1.49). The difference in response rate was $22.3 \%$ (95\% confidence interval $17.1 \%$ to $27.6 \%$ ) and the number needed to treat for relief of symptoms during six months of treatment was 4.48 (95\% confidence interval 3.62 to 5.85 ).

Conclusion This meta-analysis of individual patient data provides evidence that naftidrofuryl has a clinically meaningful effect compared with placebo in improving walking distance in patients with intermittent claudication.

\section{INTRODUCTION}

Peripheral arterial occlusive disease, with or without symptoms, occurs in about $12 \%$ of adults in the Western world and in over $20 \%$ of adults aged over
$70 .^{1-6}$ The disease is also prevalent in developing countries. $^{7-9}$

Intermittent claudication, a hallmark of symptomatic peripheral arterial occlusive disease, occurs in about $25 \%$ of patients with peripheral arterial disease. The symptoms are leg pain while walking leading to important physical limitations, and reduction in quality of life. Patients with intermittent claudication are at increased risk of cardiovascular morbidity and mortality. ${ }^{10-12}$ Prevalence and severity of the disease increase with age and the presence of cardiovascular risk factors, mainly smoking and diabetes..$^{1314}$

Treatment for intermittent claudication aims to slow the progression of atherosclerosis, reduce cardiovascular and cerebrovascular morbidity and mortality, relieve symptoms (improvement of walking distance), and improve quality of life.

Secondary prevention of cardiovascular disease is an important part of the treatment plan. ${ }^{15-20}$ Improvement of symptoms can be achieved by supervised exercise ${ }^{21}$ smoking cessation, ${ }^{22-27}$ and drug treatment. Along with their cardiovascular protective effect, statins improve the distance walked. ${ }^{17}$ In a recent Cochrane review based on six randomised controlled trials, cilostazol (100 mg twice daily), a phosphodiesterase inhibitor, was moderately effective at improving walking distance in patients with intermittent claudication compared with placebo. ${ }^{28}$ For more than 30 years vasoactive drugs have been proposed for use in patients with intermittent claudication to improve walking distance, either the pain-free walking distance or the maximum walking distance. Robust data on the efficacy of most vasoactive drugs have been lacking. ${ }^{29}$ Although these drugs have been well promoted, their use has been controversial. Several meta-analyses of this drug class were carried out $\mathrm{t}^{30-33}$ and concluded that the effects of vasoactive drugs on the symptoms of intermittent claudication were modest. Since this drug class consists of products with heterogeneous mechanisms of action, differences in clinical efficacy are possible.

In a systematic review based on published aggregated data on vasoactive drugs, naftidrofuryl was singled out as a potential candidate for further research. ${ }^{34}$ This drug has been marketed since 1968 
on the basis of presumed vascular, antiplatelet, and intracellular actions. ${ }^{35}$ Numerous reviews on naftidrofuryl have been published ${ }^{36-42}$ and the drug is mentioned in European and transatlantic guidelines, ${ }^{4344}$ but no final judgment on its efficacy has been made by the modern approach of systematic meta-analysis. In the one systematic review on naftidrofuryl, five of six eligible randomised clinical trials showed a significant, albeit modest, effect. ${ }^{34}$ Owing to heterogeneity ${ }^{45}$ among the study results, however, the authors refrained from classic metaanalysis of published aggregated data. These methodological problems could be overcome only by a metaanalysis of individual patient data. We carried out a meta-analysis within the framework of the Cochrane Collaboration to assess the feasibility of engaging in head to head trials with cilastazol, or on top of trials with statins.

We used individual patient data to determine the efficacy and safety of oral naftidrofuryl compared with placebo in improving walking distance in patients with intermittent claudication.

\section{METHODS}

We searched the Cochrane trials registers, Medline (1966 onwards), Embase (1980 onwards), Science Citation Index, and International Pharmaceutical Abstracts for trials of oral naftidrofuryl for the treatment of intermittent claudication, and we hand searched journals and conference proceedings. Relevant bibliographies were checked and we contacted the authors of identified trials and Merck Laboratories, registration holder of naftidrofuryl, for any unpublished data. No language restrictions were applied.

\section{Selection criteria and validity assessment}

We selected randomised placebo controlled clinical trials of patients with intermittent claudication who received oral naftidrofuryl $600 \mathrm{mg}$ daily or placebo and that had pain-free walking distance as the primary outcome, defined as the distance walked during a standardised exercise test before the onset of pain. The secondary outcome was the maximum walking distance.

We assessed the selected trials for quality of trial performance and reporting. Internal validity was assessed by checking for appropriateness of the randomisation process, the double blinding process, and the handling of dropouts and withdrawals by intervention group. ${ }^{46}$

We used three additional disease specific quality criteria: sufficient reporting of variability of results (disregarded when individual patient data were available), minimum sample size of 30 participants, and minimum study duration of three months. ${ }^{47}$

\section{Extraction of individual patient data}

The principal investigators and the marketing authorisation holder of naftidrofuryl (Merck Darmstadt) were contacted for the original individual patient data. Data from secondary databases were checked against the

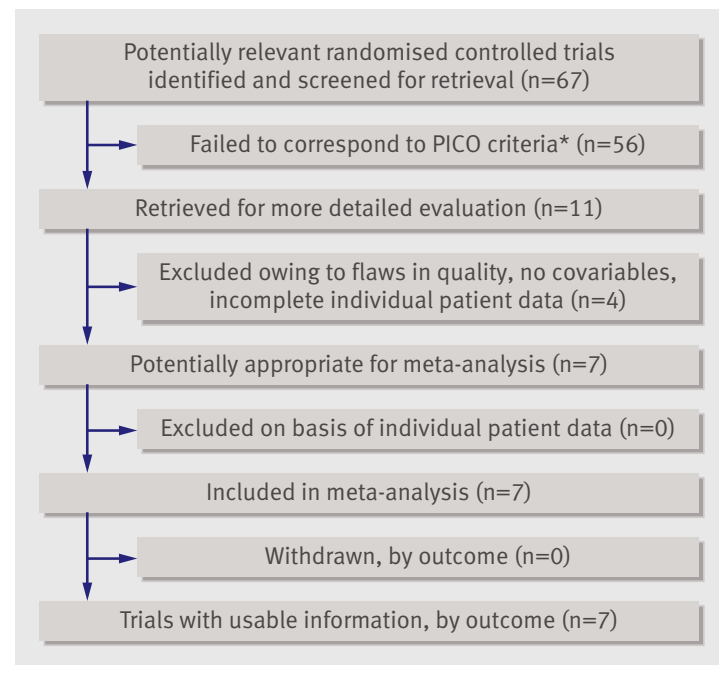

Flow of papers through trial. *Patients with intermittent claudication (Fontaine stage II), intervention oral naftidrofuryl, comparator oral placebo, and outcome walking distance measured by standardised test

original case report forms. All randomised patients with a case report form or database record were entered in the pooled database (intention to treat principle). We retrieved those patients lost to follow-up or excluded in the published per protocol analyses and included them in the database of individual patient data.

\section{Quantitative data synthesis}

The research team agreed a statistical analysis plan conforming to regulatory guidelines. ${ }^{48}$ Treatment codes were unknown and only provided at the end of the analysis process. The main analysis was based on intention to treat, in which all randomised patients were considered. To enable comparison with the published results, for each trial we identified those patients providing data in the original publication, whether they had completed the study and been compliant with the regimen (per protocol) or had at least one value after baseline (restricted intention to treat).

We carried out an exploratory stepwise regression of final walking distance to determine adjustment for covariables. In this model, baseline walking distance was singled out as the essential mediator of all other baseline variables. Hence the model using individual patient data was adjusted for baseline walking distance only.

For missing data on main end points we attributed the worst case value in patients for whom the trial was interrupted early for reasons related to peripheral arterial occlusive disease (aggravation, admission to hospital, or surgery). In all other randomised patients for whom the trial was interrupted early, we used the last observation carried forward. For the purposes of sensitivity assessment, we carried out an alternative analysis using summary statistics when at least two intermediate post-baseline observations were available. 
We checked for missing data on covariables at random and then imputed data using a systematic full information maximum likelihood technique. ${ }^{49}$ The main end point for each patient was the relative improvement in pain-free walking distance - that is, the final pain-free walking distance divided by the baseline pain-free walking distance. For all participants we used the geometric mean percentage of change from baseline, with $95 \%$ confidence intervals. We measured the treatment effect using the ratio of the relative improvement in pain-free walking distance after use of naftidrofuryl compared with placebo. A similar approach was used for the secondary outcome of maximal walking distance, for trials in which this outcome was available.

Effect was also expressed in an analysis of responders -those patients whose pain-free walking distance improved by at least $50 \%$ from baseline. We calculated the difference in success rates between naftidrofuryl and placebo, the number needed to treat, the relative benefit, and the odds ratio.

We carried out analyses using one stage and two stage approaches. In the one stage approach the database is considered as one dataset. In the two stage approach the aggregated results of each study are pooled and then analysed. ${ }^{50}$

In the one stage approach for relative improvement we used the multilevel (patient level and trial level) general linear mixed model allowing analysis of covariance $^{51}$ by considering random treatment effects and fixed study effects and adjusting for baseline walking distance. In the two stage approach we calculated aggregate estimates of relative improvement for each treatment group within each study, then used a conventional random model of DerSimonian and Laird. ${ }^{52}$

In the one stage approach for the responder analysis we used the mixed model for binary data ${ }^{53}$ and for the two stage approach we used the random model of DerSimonian and Laird. ${ }^{52}$

We set the $\alpha$ level of error for significance of the treatment effect at 0.01 . The confidence intervals were presented at the $95 \%$ level. We tested stability of the results using all trials, excluding and including debatable trials, by comparing the results of full intention to treat with restricted intention to treat and per protocol analysis and by comparing the last observation carried forward with missing data allocation based on summary statistics.

Safety

We assessed the safety of oral naftidrofuryl by reviewing the reports of adverse drug reactions in the randomised controlled clinical trials, by retrieving published case reports, and by analysing the latest periodic reports on safety from Merck Laboratories.

\section{RESULTS}

Of the studies retrieved, 11 randomised controlled trials conformed to the PICO criteria (participants, intervention, comparator, and outcome) and were selected (table 1). Four trials were excluded after quality assessment because of problems with internal validity $^{\mathrm{w1} \mathrm{w}^{\mathrm{w}} \mathrm{w}}$ or absence of both good quality individual patient data and covariables. ${ }^{\text {wl-w4 }}$ Two trials had design flaws. ${ }^{\mathrm{w} 1 \mathrm{w} 4}$ Individual patient data were not available for the other two studies. ${ }^{\text {w2 w3 }}$ Covariables were completely or partially absent in these four trials. ${ }^{\mathrm{wl}-\mathrm{w} 4}$ Seven studies were included in the analysis (table 2 and figure). ${ }^{\text {w-w11 }}$

In a previous meta-analysis based on published aggregate data ${ }^{34}$ one study ${ }^{\mathrm{w} 9}$ was excluded because it showed no data on variability of the results. That problem was solved here by using individual patient data. The study also had poor internal validity, and over $70 \%$ of the patients had a baseline pain-free

Table 1 Assessment of trial quality and availability of individual patient data in trials included in meta-analysis of naftidrofuryl compared with placebo for treatment of intermittent claudication

\begin{tabular}{|c|c|c|c|c|c|c|c|}
\hline \multirow[b]{2}{*}{ References } & \multicolumn{3}{|c|}{ Disease specific criteria* } & \multirow{2}{*}{$\begin{array}{l}\text { General criteria for internal } \\
\text { validity }\end{array}$} & \multicolumn{2}{|c|}{ Individual patient data } & \multirow{2}{*}{$\begin{array}{l}\text { Overall quality } \\
\text { grade } f\end{array}$} \\
\hline & Sample size & Duration & Variability $\dagger$ & & Outcome & Covariables & \\
\hline \multicolumn{8}{|l|}{ Excluded studies: } \\
\hline Ruckley et al $1978^{\mathrm{w} 1}$ & 0 & 1 & 2 & 2 & Incomplete & Not available & C \\
\hline Pohle et al $1979^{\text {w2 }}$ & 1 & 1 & 0 & 1 & Incomplete & Not available & $\mathrm{C}$ \\
\hline Clyne et al $1980^{\text {w3 }}$ & 0 & 0 & 0 & 1 & Incomplete & Not available & C \\
\hline Karnik et al $1988^{\text {w4 }}$ & 1 & 1 & 2 & 2 & Incomplete & Not available & C \\
\hline \multicolumn{8}{|l|}{ Included studies: } \\
\hline Maass et al $1984^{\text {w5 }}$ & 0 & 1 & 0 & 1 & Available & Available & B \\
\hline Adhoute et al $1990^{\text {w6 }}$ & 0 & 0 & 0 & 1 & Available & Available & B \\
\hline $\begin{array}{l}\text { Kriessman and Neiss } \\
1988^{\text {w7 }}\end{array}$ & 0 & 1 & 0 & 1 & Available & Available & B \\
\hline Adhoute et al $1990^{\text {w8 }}$ & 0 & 0 & 0 & 1 & Available & Available & B \\
\hline Moody et al $1994^{\mathrm{w} 9}$ & 0 & 0 & 2 & 2 & Available & Available & B \\
\hline Boccalon et al $2001^{\mathrm{w} 10}$ & 0 & 0 & 1 & 0 & Available & Available & B \\
\hline Kieffer et al $2001^{\text {w11 }}$ & 0 & 0 & 1 & 0 & Available & Available & $B$ \\
\hline
\end{tabular}

*Flaws: 0, none; 1, minor; 2, major.

†Risk of bias: grade A, no or low risk; grade B, moderate risk; and grade C, high risk.

$\ddagger$ No or incomplete reporting of variability is a major flaw but was disregarded in evaluation when individual patient data were available. 
Table 2 |Sample size, duration of trials, dosages of naftidrofuryl or placebo, and aspirin use in studies included in meta-analysis on efficacy of naftidrofuryl compared with placebo for treatment of intermittent claudication

\begin{tabular}{|c|c|c|c|c|c|}
\hline \multirow[b]{2}{*}{ Studies } & \multicolumn{2}{|c|}{ Sample size } & \multirow[b]{2}{*}{ Duration (months) } & \multirow[b]{2}{*}{ Drug dosage (naftidrofuryl or placebo) } & \multirow[b]{2}{*}{ No (\%) using aspirin } \\
\hline & Individual patient data* & Per protocol & & & \\
\hline Maass et al ${ }^{\mathrm{w} 5}$ & 142 & 104 & 3 & $200 \mathrm{mg}$ three times daily & $94(66)$ \\
\hline Adhoute et al ${ }^{\mathrm{w} 6}$ & 146 & 118 & 6 & $200 \mathrm{mg}$ three times daily & $88(60)$ \\
\hline Kriessman and Neiss $^{\text {w7 }}$ & 235 & 136 & 3 & 316.5 mg twice daily & $31(78) \dagger$ \\
\hline Adhoute et al ${ }^{\mathrm{w} 8}$ & 182 & 94 & 6 & $316.5 \mathrm{mg}$ twice daily & $109(60)$ \\
\hline Moody et al ${ }^{\mathrm{w} 9}$ & 183 & 170 & 6 & 316.5 mg twice daily & $115(63)$ \\
\hline Boccalon et alw10 & 182 & 122 & 12 & $200 \mathrm{mg}$ three times daily & $117(65)$ \\
\hline Kieffer et al ${ }^{\mathrm{w} 11}$ & 196 & 152 & 8 & $200 \mathrm{mg}$ three times daily & $127(65)$ \\
\hline $\mathrm{All}^{\text {w5-w11 }}$ & 1266 & 896 & 6.3 & - & $681(64)$ \\
\hline
\end{tabular}

*Intention to treat.

†Accurate data were available for 1071 patients; study by Kriessmann and Neiss ${ }^{\text {w7 }}$ had reliable data on aspirin use in only 40 patients (data were missing for 195 patients).

walking distance of less than $100 \mathrm{~m}$. Therefore the study was classified as supportive, disregarded in the main analysis of individual patient data, but considered in a sensitivity analysis.

Another study ${ }^{\mathrm{w} 10}$ was of good quality, except that the variability of the results was not well reported, which again could be corrected by using individual patient data. That study, however, used a non-conventional method for measuring walking distance (peripheral arterial disease holter control or PADHOC). This method is claimed to represent a more physiological walking distance but results in higher baseline and final values for walking distance. The trial was included but the data were expressed as relative improvement and not in absolute terms (metres gained). In addition, the impact of this trial was evaluated in the sensitivity analysis.

The integrity of the data collected from secondary databases was randomly checked against the original case report forms (www.sqconline.com/mil-std-105. $\mathrm{html}$ ), providing an estimate of at least 99\% for data reliability. Those patients who contributed to the original published analyses were identified from the database by using the individual patient data (restricted intention to treat ${ }^{\mathrm{w} 9-\mathrm{w} 11}$ and per protocol $\left.{ }^{\mathrm{w} 5-\mathrm{w} 8}\right)$. These data enabled confirmation of the concordance between the results of this subset in our database and the published results.

Sixty five patients had only one baseline value for pain-free walking distance, 189 for whom the trial was interrupted had at least one value for walking distance, and 1012 completed the trial and had data at each follow-up. However, in keeping with a full intention to treat analysis all patients lost to follow-up were included. A last observed carried forward strategy was used, and sensitivity analysis was carried out by comparing with results based on two other missing data allocation techniques (summary statistics and mixed model analysis).

Overall, 7.8\% of covariables were found to be missing at random and were allocated by a full information maximum likelihood procedure. Data were missing for smoking $(n=11)$, systolic blood pressure $(n=10)$, diabetes $(n=15)$, hyperlipidaemia $(n=12)$, exercise or sedentary lifestyle $(n=9)$, angina pectoris $(\mathrm{n}=11)$, height $(\mathrm{n}=2)$, and weight $(\mathrm{n}=1)$. Missing data were uniformly distributed across all variables and cases and were considered as missing at random check.

\section{Publication bias}

Formal checks for publication bias were problematic because of the small variation in sample size of the studies, ranging from 142 to 235. In statistical terms publication bias would need to be interpreted by the square root of the sample size $(11.9,15.3)$, which is a small range. Hence, funnel plots based on detection of a positive monotonic relation between efficacy and sample size are of limited relevance.

No traces to unpublished studies were found, despite a search of trial registers and the reference lists of relevant articles (snowballing).

\section{Study characteristics}

In total, 1266 patients (626 placebo, 640 naftidrofuryl) from seven studies constituted the individual patient data in the database: Maass et al $(\mathrm{n}=142),{ }^{\mathrm{w} 5}$ Adhoute et al (146) ${ }^{\text {w6 }}$ Kriessman and Neiss (235), ${ }^{\text {w7 }}$ Adhoute et al (182), ${ }^{\text {w8 }}$ Moody et al (183), ${ }^{\text {w9 }}$ Boccalon et al (182), ${ }^{\text {w10 }}$ and Kieffer et al (196). ${ }^{\text {w11 }}$ Table 3 summarises the characteristics of the sample.

The mean age of the patients was 62.41 years and the body mass index was 24.92 . They had mildly increased systolic blood pressures $(148.96 \mathrm{~mm} \mathrm{Hg})$ and as expected they had a decreased ankle brachial index, of 0.65. Just over one sixth of the patients were women. About $50 \%$ were smokers, $56 \%$ had hypertension, $13 \%$ had diabetes, 38\% had hyperlipidaemia, 43\% had a sedentary lifestyle, and 11\% had angina.

There was heterogeneity between studies but the two treatment arms were well matched for baseline walking distance (geometric mean $140.62 \mathrm{~m}$ for naftidrofuryl $v$ $140.26 \mathrm{~m}$ for placebo) and for covariables within each study as well as in the full set. Stepwise linear regression of the final walking distance as a dependent variable revealed that baseline walking distance was the key predictor $\left(\mathrm{R}^{2}=0.479\right)$, not the covariables.

In total, 896 patients $(71 \%)$ had data available for the final assessment, $305(24 \%)$ had at least one assessment after baseline, and $65(5 \%)$ had no outcome data, only a randomisation code. 
Table 3|Samples' characteristics by trial arm of studies on efficacy of naftidrofuryl compared with placebo for treatment of intermittent claudication. Values are numbers (percentages) of patients unless specified otherwise

\begin{tabular}{|c|c|c|c|}
\hline Variables & Overall $(n=1266)$ & Naftidrofuryl $(n=640)$ & Placebo $(n=626)$ \\
\hline Mean (SD) age (years) & $62.41(9.48)$ & $62.45(9.39)$ & $62.36(9.58)$ \\
\hline Mean (SD) body mass index & $24.92(3.26)$ & $24.93(3.57)$ & $24.92(2.91)$ \\
\hline Mean (SD) systolic blood pressure $(\mathrm{mm} \mathrm{Hg})$ & $148.96(20.92)$ & $148.63(20.67)$ & $149.3(21.18)$ \\
\hline Mean (SD) ankle brachial index & $0.65(0.17)$ & $0.64(0.17)$ & $0.64(0.17)$ \\
\hline Mean (SD) duration of peripheral arterial disease (years) & $3.35(3.41)$ & $3.42(3.52)$ & $3.29(3.3)$ \\
\hline Geometric mean $(95 \% \mathrm{Cl})$ walking distance $(\mathrm{m})$ & 140.44 (135.00 to 146.11$)$ & $140.62(133.07$ to 148.61$)$ & $140.26(132.52$ to 148.45$)$ \\
\hline Women & $222(17.5)$ & $121(18.9)$ & $101(16.1)$ \\
\hline Obesity & $592(46.8)$ & $295(46.1)$ & $297(47.4)$ \\
\hline Current smoker & $630(49.8)$ & $331(51.7)$ & $299(47.8)$ \\
\hline Hypertension & $710(56.1)$ & $372(58.1)$ & $338(54)$ \\
\hline Type 2 diabetes & $170(13.4)$ & $96(15)$ & $74(11.8)$ \\
\hline Hyperlipidaemia & $486(38.4)$ & $242(37.8)$ & 244 (39) \\
\hline Sedentary & $541(42.7)$ & $278(43.4)$ & 262 (41.9) \\
\hline Angina & $142(11.2)$ & $82(12.8)$ & $60(9.6)$ \\
\hline
\end{tabular}

\section{Analysis}

The main analysis was carried out on an intention to treat basis on all patients in the six trials (1083 patients). One study, by Moody et al, ${ }^{\text {w9 }}$ was excluded.

In the one stage approach the relative improvement in pain-free walking distance after use of naftidrofuryl compared with placebo was 1.37 (95\% confidence interval 1.27 to $1.49 ; \mathrm{P}<0.001)$. The analysis of responders (table 4 ) showed that $30.2 \%$ responded to placebo and $54.7 \%$ to naftidrofuryl. The absolute difference in response rate was $22.3 \%(17.1 \%$ to $27.6 \%)$. The number needed to treat was $4.48(95 \%$ confidence interval 3.62 to 5.85 ).

In the analysis of the secondary outcome, maximum walking distance was not measured in all studies, and only in a subset of patients in some ( $\mathrm{n}=968$, six studies). The relative improvement in maximum walking distance was $1.40(95 \%$ confidence interval 1.19 to 1.63), and the absolute difference in response rates was $23.9 \%(15.7 \%$ to $32.1 \%)$.

The estimates for treatment effect using the one stage approach were more conservative than those using the

\begin{tabular}{|c|c|c|}
\hline Relative improvement analysis & One stage approach & Two stage approach \\
\hline $\begin{array}{l}\text { Ratio of relative improvement in pain- } \\
\text { free walking distance }(95 \% \mathrm{Cl})^{\star}\end{array}$ & 1.37 (1.27 to 1.49$)$ & $1.38(1.24$ to 1.56$)$ \\
\hline $\begin{array}{l}\text { Ratio of relative improvement in } \\
\text { maximal walking distance }(95 \% \mathrm{Cl}) \dagger\end{array}$ & 1.40 (1.19 to 1.63$)$ & $1.38(1.18$ to 1.61$)$ \\
\hline \multicolumn{3}{|l|}{ Responder analysis: } \\
\hline $\begin{array}{l}\text { Absolute difference in response } \\
\text { rates }(95 \% \mathrm{Cl})\end{array}$ & $22.3(17.1$ to 27.6$)$ & $24.8(12.2$ to 37.4$)$ \\
\hline Number needed to treat $(95 \% \mathrm{Cl})$ & 4.48 (3.62 to 5.85$)$ & $4.03(2.51$ to 8.19$)$ \\
\hline Relative benefit $(95 \% \mathrm{Cl})$ & 1.75 (1.50 to 2.03$)$ & 1.84 (1.36 to 2.45$)$ \\
\hline Odds ratio $(95 \% \mathrm{Cl})$ & 2.65 (2.10 to 3.37$)$ & $2.90(1.70$ to 4.94$)$ \\
\hline
\end{tabular}

* Ratio of final pain-free walking distance over baseline pain-free walking distance after use of naftidrofuryl compared with placebo.

†Ratio of final maximal walking distance over baseline maximal walking distance after use of naftidrofuryl compared with placebo. two stage approach, but more precise (tighter confidence intervals, table 4).

In the two stage approach the heterogeneity between studies was significant $(\mathrm{P}=0.001)$. Baseline handicap did not, however, significantly affect the difference in relative improvement (mixed model, $\mathrm{P}>0.05$ ), which remained constant for both treatment arms except for the larger initial walking distance, where the placebo effect seemed to decrease. Heterogeneity ${ }^{45}$ was taken into account in the one stage approach by multilevel techniques and in the two stage approach by the random model of DerSimonian and Laird.

In the sensitivity analysis (table 5) the addition of the study by Moody et al ${ }^{\mathrm{w} 9}$ resulted in a relative improvement after use of naftidrofuryl compared with placebo of 1.37 (95\% confidence interval 1.28 to 1.48$)$. When the study by Boccalon et $\mathrm{al}^{\mathrm{w} 10}$ was eliminated the ratio was 1.31 (1.24 to 1.39). For the per protocol sample $(n=726)$ the value was 1.42 (1.22 to 1.65$)$. The ratio for the last observation carried forward compared with the summary statistics was 1.37 (1.28 to 1.48$)$ for naftidrofuryl compared with 1.52 (1.44 to 1.62 ) for placebo. These results all remained statistically significant.

A sensitivity analysis on the impact of the four excluded trials, ${ }^{\text {w1-w4 }}$ based on effect sizes, indicated that their inclusion would have a neutral to inflating effect on the results, without changing the direction of the conclusions.

\section{Safety}

No serious drug related events or fatalities were reported in the reports of the clinical trials. The few published case reports on adverse effects of oral naftidrofuryl pertain to liver injury, ${ }^{54-56}$ calcium oxalate crystalluria with evolution to lithiasis, ${ }^{575}$ and severe rhythm and conduction abnormalities due to massive poisoning. ${ }^{59}$ A larger number of published cases of severe events relate to the historical parenteral use of naftidrofuryl.

In the latest safety update from Merck Laboratories, the maximum incidence at 95\% confidence of drug 
$\overline{\text { Table } 5 \text { | Sensitivity analysis (one stage approach) using individual patient data on efficacy of }}$ naftidrofuryl compared with placebo for treatment of intermittent claudication

\begin{tabular}{|c|c|c|}
\hline Analysis & Sample size & Mean relative improvement* $(95 \% \mathrm{Cl})$ \\
\hline $\begin{array}{l}\text { Main analysis (all studies minus Moody } \\
\text { et } \mathrm{al}^{\mathrm{w}}{ }^{2} \text { ) }\end{array}$ & 1083 & 1.37 (1.27 to 1.48$)$ \\
\hline \multicolumn{3}{|l|}{ Sensitivity analysis: } \\
\hline All studies ${ }^{w 5-w 11}$ & 1266 & $1.37(1.28$ to 1.48$)$ \\
\hline All studies minus Boccalon et al ${ }^{w 10}$ & 1084 & 1.31 (1.24 to 1.39$)$ \\
\hline $\begin{array}{l}\text { All studies minus Moody et al }{ }^{\mathrm{w} 9} \text { and } \\
\text { Boccalon et } \mathrm{al}^{\mathrm{w} 10}\end{array}$ & 901 & $1.32(1.24$ to 1.40$)$ \\
\hline Per protocol & 726 & $1.42(1.22$ to 1.65$)$ \\
\hline
\end{tabular}

* Relative improvement in pain-free walking distance after use of naftidrofuryl compared with placebo.

related adverse events with oral naftidrofuryl was 0.89 per 100000 patient treatment years. These events essentially consisted of gastric disorders, with an absolute difference in risk of $2.85 \%(0.78 \%$ to $4.91 \%)$ compared with placebo and relative risk of 1.75 (1.25 to $2.45)$. No other specific organ disorder was of clinically relevant incidence. A limited number of neurological, cardiovascular, and skin adverse reactions were reported, but their incidence was no different than with placebo.

\section{DISCUSSION}

In patients with intermittent claudication behavioural and pharmacological measures to modify the cardiovascular risk profile are of prime importance. Symptomatic treatment by improving the walking distance can have a clinically relevant impact on functional capability and quality of life. This can be achieved by behavioural measures (exercise and smoking cessation) as well as drug treatment.

Evidence on the efficacy of drugs to achieve relief of symptoms in intermittent claudication is available for statins and cilostazol. This review using individual patient data provides statistical evidence that naftidrofuryl, picked out of the controversial class of vasoactive drugs, has a moderate clinically meaningful effect by improving walking distance in patients with intermittent claudication. Patients in the naftidrofuryl group walked $37 \%$ further than patients in the placebo group. The number of patients responding to naftidrofuryl (improvement by more than 50\%) was $22 \%$ higher than

\section{WHAT IS ALREADY KNOWN ON THIS TOPIC}

Exercise training and statins have proved beneficial in the symptomatic treatment of intermittent claudication

The use of vasoactive drugs has always been controversial because of poor and unconfirmed evidence of clinical efficacy

\section{WHAT THIS STUDY ADDS}

Naftidrofuryl is moderately effective at improving walking distance in patients with intermittent claudication

Head to head trials with cilostazol and on top of trials with statins can now be done with assessment of ratio of benefit to risk, quality of life, and cost effectiveness

Older products should undergo a thorough review of their ratio of benefit to risk the number responding to placebo. Once superiority over placebo is proved, the way is open for head to head trials with active drugs for symptomatic treatment of intermittent claudication.

Quality of life measures may add robustness to the efficacy data. ${ }^{6061}$ The tolerance and safety profiles of oral naftidrofuryl are acceptable. The current market price for naftidrofuryl at a dosage of $200 \mathrm{mg}$ three times daily is $€ 1(£ 0.90 ; \$ 1.26)$.

The use of oral naftidrofuryl to treat intermittent claudication could be recommended in patients who still have symptoms after maximal secondary prevention measures, including exercise therapy (if feasible), or after revascularisation (if indicated), with re-evaluation after three to six months. Within the indication of symptomatic treatment, its use should be evaluated against cilostazol and statins.

The authors believe that the results of this metaanalysis on the efficacy of naftidrofuryl using individual patient data can be trusted. Access to the data, however, depended on the goodwill of Merck Darmstadt, the marketing authorisation holder. We found references and full text of identified studies in the medical literature, but for individual patient data we had to rely on the permission of Merck Laboratories, as data were not readily available from the principal investigators. The company, who funded most of the studies in this review, provided the data without preliminary conditions. The meta-analysis was funded by our university and not by Merck Laboratories, as directed by the Cochrane Collaboration. The data were critically verified and analysed. Despite checks on the integrity of the data some uncertainty remains about the authenticity of the original data, as some studies date from before the period when rigorous checks for good clinical practice were implemented. However our comparison of the per protocol and restricted intention to treat data in our database with the published results were reassuring.

In this study we exploited several advantages of using individual patient data: standardisation of outcome choice (pain-free walking distance), standardisation of expressing the outcome (geometric mean of relative improvement) across all studies, application of powerful analytical methods taking into account heterogeneity, and full intention to treat analysis.

We found that it is difficult but not impossible to collect data from randomised clinical trials and individual patients, even for older products with studies done decades ago. For newly developed medicines, however, shared ownership or unrestricted access to individual patient data from drug related randomised controlled trials should be possible for the principal investigators of the trials, legal statistical supervisors, regulatory authorities, and researchers producing evidence based summaries of existing knowledge, with due consideration of patient privacy.

This paper is based on a Cochrane review published in the Cochrane Library on 16 April, issue 2, 2008.

Contributors: TDeB contributed to the planning, literature search, data collection, quality assessment of the studies, data extraction, data 
analysis, data interpretation, and writing of the report. All steps were critically reviewed and discussed with all authors. RVS contributed to the planning, literature search, quality assessment, data interpretation, and writing of the report. He is guarantor. PL contributed to the statistical analysis plan and data analysis. LVB contributed to the planning, quality assessment, and writing of the report.

Funding: This study was funded by Ghent University, Belgium. The Cochrane Collaboration is supported by the Chief Scientist Office, Health Department, the Scottish Executive, UK.

Competing interests: PL has carried out statistical consultancy for several pharmaceutical companies, including Merck

Ethical approval: Not required.

1 Hiatt WR, Hoag S, Hamman RF. Effect of diagnostic criteria on the prevalence of peripheral arterial disease. The San Luis Valley Diabetes Study. Circulation 1995;91:1472-9.

2 Fowkes FG, Housley E, Cawood EH, Macintyre CC, Ruckley CV, Prescott RJ. Edinburgh Artery Study: prevalence of asymptomatic and symptomatic peripheral arterial disease in the general population. Int J Epidemiol 1991;20:384-92.

3 Sprynger M, Fassotte C, Verhaeghe R. The ankle-brachial pressure index and a standardized questionnaire are easy and useful tools to detect peripheral arterial disease in non-claudicating patients at high risk. Int Angiol 2007;26:239-44.

4 Sigvant B, Wiberg-Hedman K, Bergqvist D, Rolandsson O, Andersson B, Persson E, et al. A population-based study of peripheral arterial disease prevalence with special focus on critical limb ischemia and sex differences. / Vasc Surg 2007;45:1185-91.

5 Dormandy JA, Rutherford RB. Management of peripheral arterial disease (PAD). TASC Working Group. TransAtlantic Inter-Society Consensus (TASC). I Vasc Surg 2000;31:S1-296.

6 Carman TL, Fernandez BB, Jr. A primary care approach to the patient with claudication. Am Fam Phys 2000;61:1027-32, 1034.

7 Li XY, Wang J, He Y, Fan L. The relation between peripheral arterial occlusive disease and cardiovascular diseases in elderly population: a cross-section study in Wanshoulu area, Beijing. Zhonghua Yi Xue Za Zhi 2003;83:1847-51.

8 Robbs JV. Atherosclerotic peripheral arterial disease in blacks-an established problem. S Afr Med J 1985;67:797-801.

9 Madiba TE, Mars M, Robbs JV. Aorto-iliac occlusive disease in the various population groups of South Africa. S Afr Med J 2001;91:785-6.

10 Coffman JD. Drug therapy: vasodilator drugs in peripheral vascular disease. N Engl/ Med 1979;300:713-7.

11 Criqui $\mathrm{MH}$, Coughlin SS, Fronek A. Noninvasively diagnosed peripheral arterial disease as a predictor of mortality: results from a prospective study. Circulation 1985;72:768-73.

12 Hooi JD, Kester AD, Stoffers HE, Rinkens PE, Knottnerus JA, van Ree JW. Asymptomatic peripheral arterial occlusive disease predicted cardiovascular morbidity and mortality in a 7-year follow-up study. Clin Epidemiol 2004:57:294-300.

13 Verhaeghe R. Epidemiology and prognosis of peripheral obliterative arteriopathy. Drugs 1998;56(suppl 3):1-10.

14 Selvin E, Erlinger TP. Prevalence of and risk factors for peripheral arterial disease in the United States: results from the National Health and Nutrition Examination Survey, 1999-2000. Circulation 2004;110:738-43.

15 Leng GC, Fowler B, Ernst E. Exercise for intermittent claudication. Cochrane Database Syst Rev 2000;(2):CD000990.

16 Mohler ER, III, Hiatt WR, Creager MA. Cholesterol reduction with atorvastatin improves walking distance in patients with peripheral arterial disease. Circulation 2003;108:1481-6.

17 Aung PP, Maxwell HG, Jepson RG, Price JF, Leng GC. Lipid-lowering for peripheral arterial disease of the lower limb. Cochrane Database Syst Rev 2007;(4):CD000123.

18 Clement DL, Debuyzere ML. How to treat hypertension in patients with peripheral artery disease. Curr Hypertens Rep 2007;9:190-5.

19 Catalano M, Born G, Peto R. Prevention of serious vascular events by aspirin amongst patients with peripheral arterial disease: randomized, double-blind trial. J Intern Med 2007;261:276-84.

20 Annemans L, Lamotte M, Clarys P, Van den Abeele E. Health economic evaluation of controlled and maintained physical exercise in the prevention of cardiovascular and other prosperity diseases. Eur J Cardiovasc Prev Rehabil 2007;14:815-24.

21 Lee HL, Mehta T, Ray B, Heng MS, McCollum PT, Chetter IC. A nonrandomised controlled trial of the clinical and cost effectiveness of a supervised exercise programme for claudication. Eur JVasc Endovasc Surg 2007;33:202-7.

22 Hankey GJ, Norman PE, Eikelboom JW. Medical treatment of peripheral arterial disease. JAMA 2006;295:547-53.

23 Gardner AW, Poehlman ET. Exercise rehabilitation programs for the treatment of claudication pain. A meta-analysis. JAMA 1995;274:975-80.
24 Gardner AW, Katzel LI, Sorkin JD, Killewich LA, Ryan A, Flinn WR, et al. Improved functional outcomes following exercise rehabilitation in patients with intermittent claudication. J Gerontol A Biol Sci Med Sci 2000;55:M570-7

25 Gardner AW, Katzel LI, Sorkin JD, Bradham DD, Hochberg MC, Flinn WR, et al. Exercise rehabilitation improves functional outcomes and peripheral circulation in patients with intermittent claudication: a randomized controlled trial. J Am Geriatr Soc 2001;49:755-62.

26 Gartenmann C, Kirchberger I, Herzig M, Baumgartner I, Saner H, Mahler F, et al. Effects of exercise training program on functional capacity and quality of life in patients with peripheral arterial occlusive disease. Evaluation of a pilot project. Vasa 2002;31:29-34.

27 Christman SK, Ahijevych K, Buckworth J. Exercise training and smoking cessation as the cornerstones of managing claudication. J Cardiovasc Nurs 2001;15:64-77.

28 Robless P, Mikhailidis DP, Stansby GP. Cilostazol for peripheral arterial disease. Cochrane Database Syst Rev 2007;(1):CD003748.

29 Moher D, Pham B, Ausejo M, Saenz A, Hood S, Barber GG. Pharmacological management of intermittent claudication: a metaanalysis of randomised trials. Drugs 2000;59:1057-70.

30 Girolami B, Bernardi E, Prins MH, Ten Cate JW, Hettiarachchi R, Prandoni $P$, et al. Treatment of intermittent claudication with physical training, smoking cessation, pentoxifylline, or nafronyl: a metaanalysis. Arch Intern Med 1999;159:337-45.

31 Hood SC, Moher D, Barber GG. Management of intermittent claudication with pentoxifylline: a meta-analysis of randomized controlled trials. CMA/1996;155:1053-9.

32 Walker GA, Mac Hannaford JC. A meta-analysis of randomized, double-blind, placebo-controlled studies of the effect of buflomedil on intermittent claudication. Fundam Clin Pharmacol 1995;9:387-94.

33 Lehert P, Riphagen FE, Gamand S. The effect of naftidrofuryl on intermittent claudication: a meta-analysis. J Cardiovasc Pharmacol 1990;16(suppl 3):S81-6.

34 De Backer TL, Vander Stichele RH, Warie HH, Bogaert MG. Oral vasoactive medication in intermittent claudication: utile or futile? EurJ Clin Pharmacol 2000;56:199-206.

35 Barradell LB, Brogden RN. Oral naftidrofuryl. A review of its pharmacology and therapeutic use in the management of periphera occlusive arterial disease. Drugs Aging 1996;8:299-322.

36 Boobis LH, Bell PR. Can drugs help patients with lower limb ischaemia? Br J Surg 1982;69(suppl):S17-23.

37 Spitzer S, Bach R, Schieffer H. Walk training and drug treatment in patients with peripheral arterial occlusive disease stage II. A review. Int Angiol 1992;11:204-10.

38 Bevan EG, Waller PC, Ramsay LE. Pharmacological approaches to the treatment of intermittent claudication. Drugs Aging 1992;2:125-36.

39 Clement DL. Medical treatment of peripheral artery occlusive disease (PAOD). Acta Chir Belg 2000;100:190-3.

40 Jacoby D, Mohler ER, III. Drug treatment of intermittent claudication. Drugs 2004;64:1657-70.

41 Goldsmith DR, Wellington K. Naftidrofuryl: a review of its use in the treatment of intermittent claudication. Drugs Aging 2005;22:967-77.

42 Heidrich $\mathrm{H}$. Vasoactive agents and prostanoids in the therapy of PAD: facts, questions, disproven assumptions. Hamostaseologie 2006;26:220-3

43 Norgren L, Hiatt WR, Dormandy JA, Nehler MR, Harris KA, Fowkes FG, et al. Inter-society consensus for the management of peripheral arterial disease (TASC II). Eur J Vasc Endovasc Surg 2007;33(suppl 1):S1-75

44 Scottish Intercollegiate Guidelines Network. Diagnosis and management of peripheral arterial disease. A national clinical guideline. Edinburgh: SIGN, 2006:1-34.

45 Fletcher J. What is heterogeneity and is it important? BMJ 2007;334:94-6.

46 Jadad AR, Moore RA, Carroll D, Jenkinson C, Reynolds DJ, Gavaghan DJ, et al. Assessing the quality of reports of randomized clinical trials: is blinding necessary? Control Clin Trials 1996;17:1-12.

47 Cameron HA, Waller PC, Ramsay LE. Drug treatment of intermittent claudication: critical analysis of the methods and findings of published clinical trials, 1965-1985. Br J Clin Pharmacol 1988;26:569-76.

48 Committee for Proprietary Medicinal Products Working Party. Note for guidance on clinical investigations of medicinal products in the treatment of chronic peripheral arterial occlusive disease. London: The European Agency for the Evaluation of Medicinal Products, Oct 2002.

49 Anderson TW. Maximum likelihood estimates for a multivariate normal distribution when some observations are missing. J Am Stat Assoc 1957;55:200-3.

50 Simmonds MC, Higgins JP, Stewart LA, Tierney JF, Clarke MJ, Thompson SG. Meta-analysis of individual patient data from randomized trials: a review of methods used in practice. Clin Trials 2005;2:209-17 
51 Higgins JP, Whitehead A, Turner RM, Omar RZ, Thompson SG. Metaanalysis of continuous outcome data from individual patients. Stat Med 2001;20:2219-41.

52 DerSimonian R, Laird N. Meta-analysis in clinical trials. Control Clin Trials 1986;7:177-88.

53 Turner RM, Omar RZ, Yang M, Goldstein H, Thompson SG. A multilevel model framework for meta-analysis of clinical trials with binary outcomes. Stat Med 2000;19:3417-32.

54 Cholongitas E, Papatheodoridis GV, Mavrogiannaki A, Manesis E. Naftidrofuryl-induced liver injury. Am J Gastroenterol 2003;98:1448-50.

55 Hebuterne X, Laugery A, Chichmanian RM, Tran A, Rampal P. Acute cytolytic hepatitis probably caused by naftidrofuryl. Gastroenterol Clin Biol 1990;14:514-5.

56 De Caestecker JS, Heading RC. Naftidrofuryl-induced acute hepatic necrosis. Postgrad Med J 1986;62:309-10.
57 Moesch C, Rince M, Daudon M, Aldigier JC, Leroux-Robert C. Renal intratubular crystallisation of calcium oxalate and naftidrofuryl oxalate. Lancet 1991;338:1219-20.

58 Moesch C, Charmes JP, Bouthier F, Leroux-Robert C. Calcium oxalate crystalluria in elderly patients and treatment with naftidrofuryl oxalate. Age Ageing 1995;24:464-7.

59 Rey JL, Marek A, Tribouilloy C, Jarry G, Slama MA, Avinee P, et al. Massive poisoning by naftidrofuryl with severe disorders of rhythm and conduction. Arch Mal Coeur Vaiss 1989;82:1467-71.

60 Muller-Buhl U, Engeser P, Klimm HD, Wiesemann A. Quality of life and objective disease criteria in patients with intermittent claudication in general practice. Fam Pract 2003;20:36-40.

61 Spengel F, Clement D, Boccalon H, Liard F, Brown T, Lehert P. Findings of the naftidrofuryl in quality of life (NIQOL) European study program. Int Angiol 2002;21:20-7.

Accepted: 19 November 2008 